\title{
ENDOCRINE MARKERS OF AGING
}

\author{
JEFFREY B. HALTER \\ Department of Internal Medicine, Geriatrics Center and Institute of Gerontology, University of Michigan and \\ Ann Arbor V.A. Medical Center, Ann Arbor, Michigan
}

\begin{abstract}
Sophisticated methods available to quantitate hormone secretion and hormone action provide opportunities to identify potential endocrine biomarkers of aging. However, there are many challenges facing the investigator who wishes to establish an endocrine biomarker. Circulating levels of many hormones fluctuate on a circadian rhythm, and a variety of other short-term fluctuations may occur. Hormone secretion is generally tightly regulated and often affected by multiple, redundant feedback mechanisms. Interpretation of circulating hormone levels must also take into account factors affecting hormone metabolism and degradation which may be influenced by age. The rate of hormone secretion and circulating hormone levels are related to the sensitivity to hormone action by feedback control mechanisms. Thus interpretation of potential circulating endocrine markers of aging must take into account changes in sensitivity to that hormone as well.
\end{abstract}

Key Words: hormones, feedback control, testosterone, insulin, growth hormone, norepinephrine, receptors

\section{INTRODUCTION}

THE ENDOCRINE system is concerned with the secretion of hormones, or chemical messengers, into the circulatory system and the subsequent action of these messengers at distant sites to regulate a number of internal homeostatic control mechanisms. Thus, aspects of the endocrine systems' function can be assessed by direct measurements of hormone levels in the circulatory system. With the development of sophisticated methods for accurate measurement of circulating hormone levels, there has been considerable opportunity to obtain such measurements in humans and a variety of animal species to determine whether hormone levels change with aging. Such measurements have provided the basis for proposing a number of endocrine hormones or neurotransmitters as biomarkers of aging. However, a number of factors must be considered in the interpretation of circulating hormone levels and their potential use as biomarkers for aging.

Studies of circulating hormones must take into account the fact that most hormones do not circulate at constant levels, but fluctuate considerably. The circulating hormone level is also a function of a dynamic equilibrium between rates of hormone secretion and hormone metabolism. Thus, a change in hormone level with age can reflect not only a change in the rate of hormone secretion, but also a change in the rate at which the

Correspondence to: J.B. Halter, University of Michigan Hospital, Division of Geriatric Medicine, 1010 Wall Street, ST1508 Turner Building, Ann Arbor, MI. 


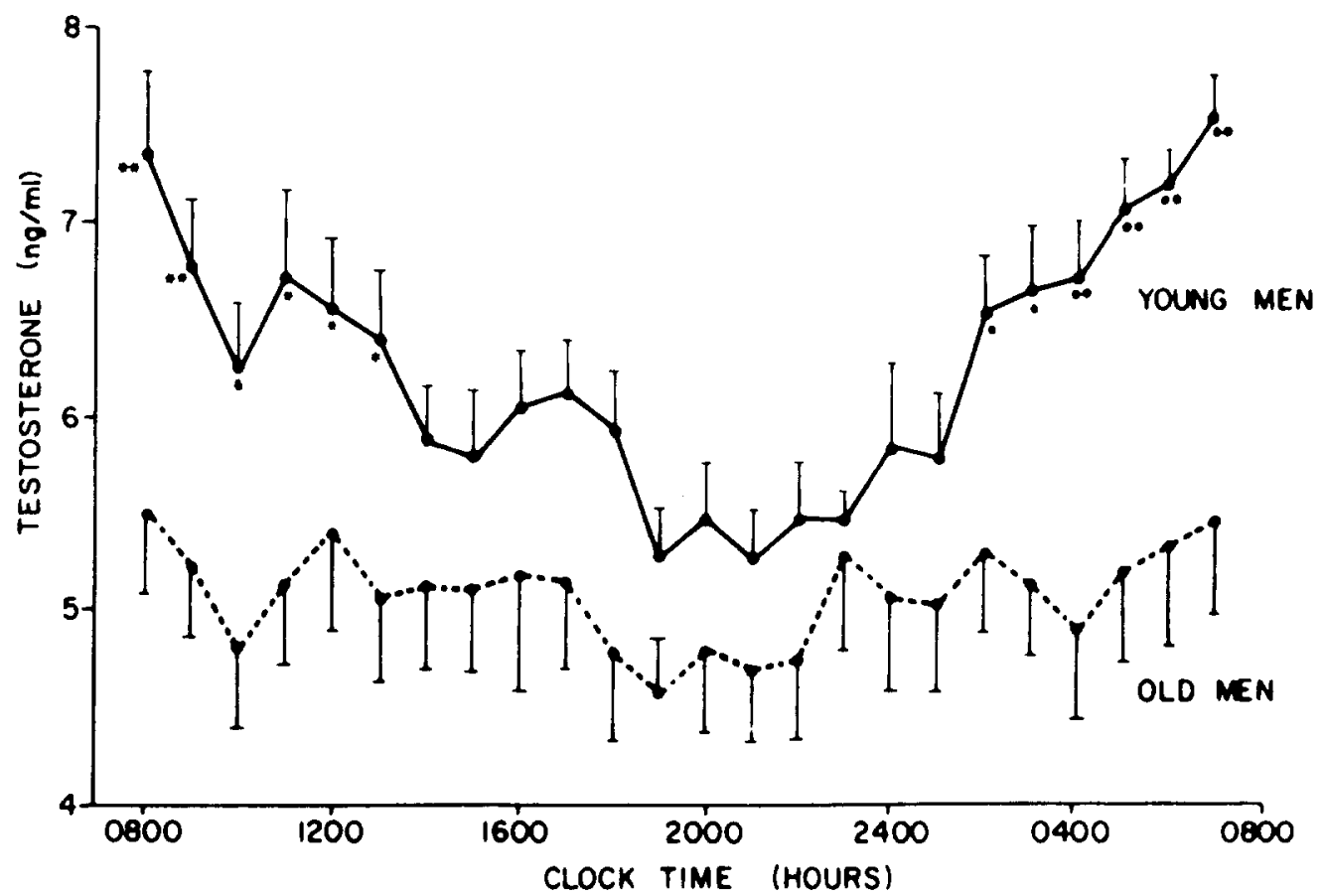

FIG. 1. Comparison of serum testosterone levels over a 24 -h period in healthy young $(n=17)$ and old $(n=12)$ men. Note the diurnal rhythm of serum testosterone that is apparent in the young. The diurnal rhythm is blunted in the elderly. Thus, there are significant age differences at night and in the morning (asterisks), but little age difference at midday (from Bremner et al., Loss of circadian rhythmicity in blood testosterone levels with aging in normal men. J. Clin. Endocrinol. Metab., 56, 1278-1281, ${ }^{\circ}$ The Endocrine Society 1983.

hormone is metabolized. Hormone secretion is tightly regulated, often by many different factors. Because hormones mediate control of internal homeostasis, they are particularly likely to change in response to environmental factors or stimuli. Thus, any apparent change in hormone levels with aging must take into account possible interactions between age and a variety of environmental factors. Finally, most hormones are regulated in the context of a feedback control system that provides for a close interaction between hormone secretion and hormone action. Thus, if resistance to hormone action develops, there is generally a feedback response to enhance hormone secretion. This article will present some examples of how each of these aspects of endocrine system function may influence the interpretation of potential biomarkers of aging.

\section{FLUCTUATIONS OF HORMONE LEVELS}

There are several different types of fluctuations of hormone levels that are recognized. The most widely known type of fluctuation is related to the circadian cycle. While it is widely recognized that hormones such as cortisol exhibit considerable circadian variation, circadian variation of other hormones also occurs and may affect interpretation of data. For example, there has been considerable controversy about whether circulating levels of testosterone decline in elderly men, based on studies which have relied on single measurements made during the day (Harman and Tsitouras, 1980; Sparrow et al., 1980; Zumoff et al., 1982; Davidson et al., 1983; Desylpere and 
Vermeulen, 1984). However, as illustrated in Figure 1, there is a diurnal rhythm of serum testosterone. The largest age-related difference occurs at night when testosterone levels are highest in the young. It is apparent that measurements made during midday are most likely to miss the age-related fall in testosterone levels (Bremner $\mathrm{et}$ al., 1983). If a decline of testosterone levels is to be used as a marker of an age-related change in testicular function, the timing and frequency of such measurements need to be seriously considered. Similarly, growth hormone levels are considerably lower in the elderly than in the young, but this difference cannot be detected if measurements are made only during the day. In young adults, growth hormone secretion occurs at night in association with deep slow wave sleep. It is this aspect of growth hormone secretion that is specifically diminished in the elderly (Prinz et al., 1983).

In addition to the diurnal swings of hormone levels that must be taken into account in interpreting aging studies, there are short-term fluctuations in hormone levels which can interfere with interpretation of results. For example, it is clear that gonadotropins are secreted in a pulsatile manner, reflecting responses to pulsatile secretion of hypothalamic peptides (Casper et al., 1979; Coquelin and Desjardins, 1982). As a result, interpretation of a single measurement of luteinizing hormone, for example, is very difficult. Characterization of the frequency and magnitude of pulsatile luteinizing hormone secretion is essential for adequate assessment of the integrity of this system. Alterations in the pattern of secretion could potentially provide a sensitive biomarker of aging of the neuroendocrine system.

\section{REGULATION OF HORMONE SECRETION}

Most hormones are part of a feedback control mechanism in which there is a single primary signal that regulates hormone secretion. However in addition to this primary signal, there are often multiple other signals which may also interact to determine the rate of hormone secretion. For example, the circulating glucose level is the most important regulator of insulin secretion. However there are multiple other hormones, neurotransmitters, and metabolites which can interact with glucose to enhance or inhibit glucose mediated insulin secretion. Thus, for interpretation of circulating insulin levels as a potential biomarker of aging, one would have to control not only for the circulating glucose level, but the whole host of other factors which can also interact. Similarly, secretion of thyroid hormone is controlled primarily by pituitary release of thyroid stimulating hormone that in turn is primarily controlled by hypothalamic secretion of thyrotropin releasing hormone. It is clear that a number of other neuroendocrine and nutritional factors can interact with this system at multiple levels which can influence hormone output. A number of early studies of plasma $T_{3}$ levels reported an age-related decline in humans. However, it was subsequently learned that regulation of thyroid hormone secretion can be greatly influenced by stressful illness and poor nutrition. When subsequent studies were done excluding elderly people who were sick or malnourished, no age-related decline in $\mathrm{T}_{3}$ levels could be documented (Olsen et al., 1978).

\section{CIRCULATING HORMONE KINETICS}

Since a number of hormones are removed rapidly from the circulation, alterations in removal mechanisms can have a considerable effect on the circulating hormone level. A number of approaches are available to assess the kinetics of hormone secretion and removal. One approach is to infuse the hormone of interest and estimate the hormone clearance rate as the infusion rate divided by the difference between the hormone level 
achieved and the basal hormone level. Because infusion of a hormone may cause physiologic effects which may influence the hormones clearance rate, a better approach is to infuse tracer amounts of radiolabelled hormone to assess its distribution and metabolism. One example of an age-related change in hormone kinetics is the observation from several laboratories that the clearance rate of insulin is diminished in healthy elderly people (Minaker et al., 1982; Reaven et al., 1982; Fink et al., 1985). Thus, plasma insulin levels in the elderly will tend to be an overestimate of acutal insulin secretion. The clearance rate of the neurotransmitter norepinephrine from the circulation also tends to be diminished in the elderly, but tracer kinetic studies have indicated that the age-related increase in plasma norepinephrine is primarily due to an increased rate of norepinephrine spillover into the circulation (Veith et al., 1986).

\section{INTERACTION OF HORMONE SECRETION AND HORMONE ACTION}

Sophisticated approaches to quantitative estimation of hormone action have been developed in recent years and have provided evidence for an age-related decline in hormone responsiveness in a number of systems. Hormone action at the cellular level initially involves interaction with a specific hormone receptor. As a result of this interaction a signal is generated which activates mechanisms that ultimately lead to the cellular response. In the case of peptide hormones and the catecholamines, which do not readily penetrate cell membranes, the receptor is located on the cell surface. The hormone receptor interaction results in activation of one or more intracellular protein kinases that phosphorylate enzymes which initiate a cascade of events resulting in the cellular response. In the case of steroid hormones, which penetrate cell membranes readily, the receptors are located intracellularly and mediate transfer of the steroid hormone to the nucleus of the cell where the hormone acts to regulate cellular protein synthesis by affecting the translation of DNA. Thus, it is apparent that alterations of hormone action in aging may involve any of these complex processes. Since hormone binding to specific receptors can be measured most readily, many studies of aging have involved such measurements. However, as more is being learned about the mediators resulting from the hormone receptor interaction, more work has focused on this aspect of hormone action.

In general, measurements of hormone binding to specific receptors have not shown consistent defects in aging even though tissue responsiveness to many hormones is diminished. Examples include reduced responsiveness to beta adrenergic stimulation in aging in the absence of consistent changes of beta adrenergic receptor number (Heinsimer and Lefkowitz, 1985) and a decline in sensitivity to the effects of insulin on glucose metabolism in the absence of a consistent change of insulin receptor number (Fink et al., 1983; Rowe et al., 1983).

An important adjunct to measurement of hormone receptors is the assessment of the cellular response to the hormone. An example is the assessment of hormone mediated regulation of the enzyme adenylcyclase by measurement of the production of cyclic AMP by the cell. An age-related impairment of production of cyclic AMP in response to beta adrenergic stimulation has been demonstrated in a number of tissues both in experimental animals and humans (Heinsimer and Lefkowitz, 1985). As illustrated in Fig. 2, there is an analogous impairment of inhibition of cyclic AMP production by platelet membranes in response to alpha adrenergic receptor stimulation with epinephrine in elderly compared to young humans (Supiano et al., 1987). 


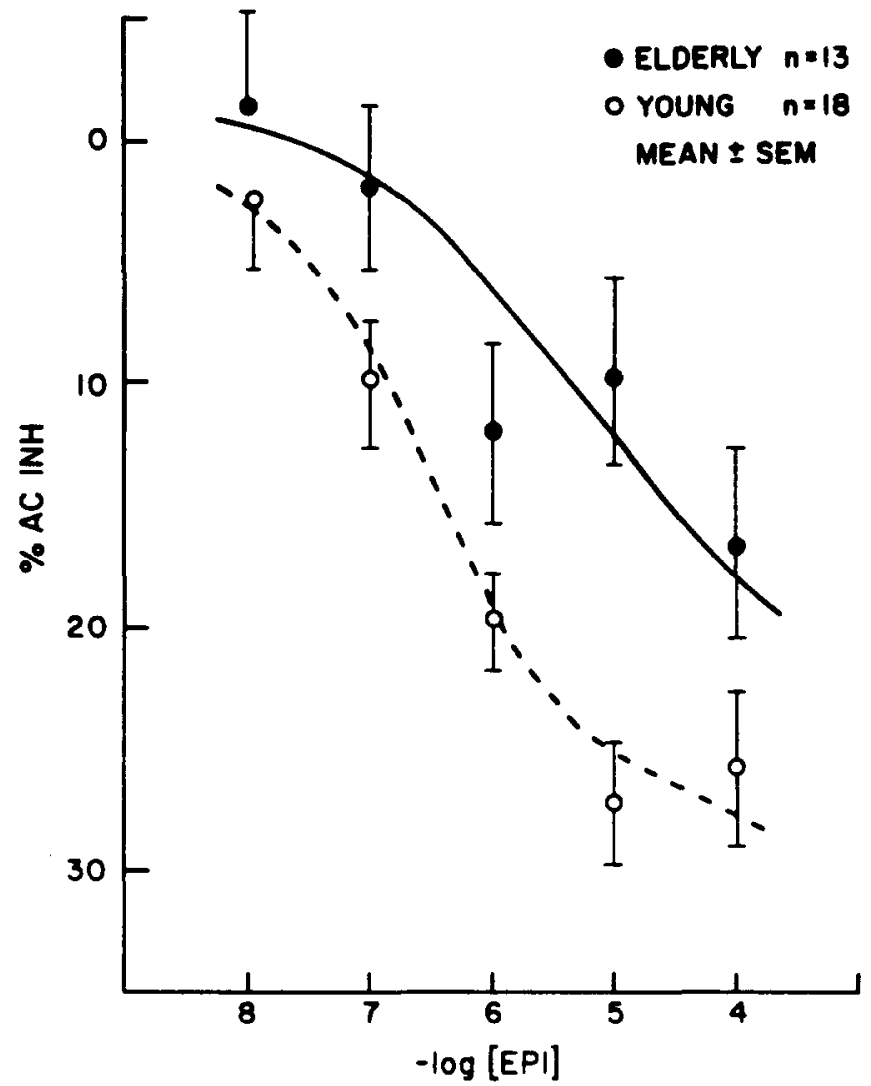

FIG. 2. Dose-response relationship for adenylate cyclase activity. The dose-response relationship in elderly and young subject groups for epinephrine (EPI) mediated inhibition of cAMP production (range $10^{-8}$ to $10^{-4} \mathrm{M}$ ) expressed as a percentage (mean $\pm \mathrm{SEM}$ ) of maximal sodium fluoride stimulated activity (\%AC INH). There is a significant age effect by ANOVA $(p<0.02)$ (from Supiano et al., Function uncoupling of the platelet alpha adrenergic receptor-adenylate cyclase complex in the elderly, J. Clin. Endocrinol. Metab., 64, 1160-1164, ${ }^{\circ}$ The Endocrine Society 1987.

The relationship between altered cellular responsiveness to hormones and the circulating hormone level also needs to be considered. When circulating levels of the hormone are elevated, this could represent an appropriate response to resistance to the action of the hormone. Alternatively, if there is a primary mechanism resulting in increased circulating hormone levels, changes in receptor function may occur which are secondary. Studies of a number of hormone-receptor systems have indicated that increased hormone levels result in downregulation of hormone responsiveness. The adrenergic system is a good example of this phenomenon. The increased circulating levels of norepinephrine observed in many studies in the elderly could represent an appropriate response to impaired cellular responsiveness to catecholamines (Linares and Halter, 1987). Alternatively, diminished adrenergic sensitivity in the elderly could represent an appropriate downregulation response to chronic elevation of catecholamine levels. 


\section{SUMMARY}

Sophisticated methods available to quantitate hormone secretion and hormone action provide a number of opportunities to identify potential endocrine biomarkers of aging. However, there are many challenges facing the investigator who wishes to establish an endocrine biomarker. By their very nature, endocrine systems are tightly regulated and often affected by multiple, redundant feedback mechanisms. Thus, careful control of such other factors must be considered when trying to tightly link a specific change in the endocrine system to the aging process. Both hormone secretion and hormone action appear to be so regulated. Thus, both of these aspects of endocrine system regulation need to be studied in order to interpret the meaning of hormone measurements and their potential relationship to aging.

\section{REFERENCES}

BREMNER, W.J., VITIELLO, M.V., and PRINZ, P.N. Loss of circadian rhythmicity in blood testosterone levels with aging in normal men. J. Clin. Endocrinol. Metab. 56, 1278-1281, 1983.

CASPER, R.F., YEN, S.S.C., and WILKES, M.M. Menopausal flushes: A neuroendocrine link with pulsatile luteinizing hormone secretion. Science 205, 823-825, 1979.

COQUELIN, A. and DESJARDINS, C. Luteinizing hormone and testosterone secretion in young and old male mice. Am. J. Physiol. (Endocrinol. Metab. 6) 243, E257-E263, 1982.

DAVIDSON, J.M., CHEN, J.J., CRAPO, L., GRAY, G.D., GREENLEAF, W.J., and CATANIA, J.A. Hormonal changes and sexual function in aging men. J. Clin. Endocrinol. Metab. 57, 71-77, 1983.

DESYLPERE, J.P. and VERMEULEN, A. Leydig cell function in normal men: Effect of age, life-style, residence, diet and activity. J. Clin. Endocrinol. Metab. 59, 955-962, 1984.

FINK, R., KOLTERMAN, O., GRIFFIN, J., and OLEFSKY, J.M. Mechanism of insulin resistance in aging. J. Clin. Invest. 71, 1523-1535, 1983.

FINK, R.I., REVERS, R.R., KOLTERMAN, O.G., and OLEFSKY, J.M. The metabolic clearance of insulin and the feedback inhibition of insulin secretion are altered with aging. Diabetes 34, 275-280, 1985.

HARMAN, S.M. and TSITOURAS, P.D. Reproductive hormones in aging men. I. Measurement of sex steroids, basal luteinizing hormone, and leydig cell response to human chorionic gonadotropin. $J$. Clin. Endocrinol. Metab. 51, 35-40, 1980.

HEINSIMER, J.A. and LEFKOWITZ, R.J. The impact of aging on adrenergic receptors: Clinical and biochemical aspects. J. Am. Geriatr. Soc. 33, 184-188, 1985.

LINARES, O.A. and HALTER, J.B. Sympathochromaffin system activity in the elderly. J. Am. Geriatr. Soc. 35, 448-453, 1987.

MINAKER, K.L., ROWE, J.W., TONINO, R., and PALLOTTA, J.A. Influence of age on clearance of insulin in man. Diabetes 31, 851-855, 1982.

OLSEN, T., LAURBERG, P., and WEEKE, J. Low serum triiodothyronine and high serum reverse triiodothyronine in old age: An effect of disease not age. J. Clin. Endocrinol. Metab. 47, 1111-1115, 1978.

PRINZ, P.N., WEITZMAN, E.D., CUNNINGHAM, G.R., and KARACAN, I. Plasma growth hormone during sleep in young and aged men. $J$. Gerontol. 38, 519-524, 1983.

REAVEN, G.M., GREENFIELD, M.S., MONDON, C.E., ROSENTHAL, M., WRIGHT, D., and REAVEN, E.P. Does insulin removal rate from plasma decline with age? Diabetes 31, 670-673, 1982.

ROWE, J.W., MINAKER, K.L., PALLOTTA, J.A., and FLIER, J.S. Characterization of the insulin resistance of aging. $J$. Clin. Invest. 71, 1582-1587, 1983.

SPARROW, D.P., BOSSE, R., and ROWE, J.W. The influence of age, alcohol consumption, and body build on gonadal function in men. J. Clin. Endocrinol. Metab. 51, 508-512, 1980.

SUPIANO, M.A., LINARES, O.A., HALTER, J.B., RENO, K.M., and ROSEN, S.G. Functional un-

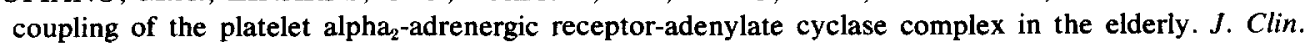
Endocrinol. Metab. 64, 1160-1164, 1987.

VEITH, R.C., FEATHERSTONE, J.A., LINARES, O.A., and HALTER, J.B. Age differences in plasma norepinephrine kinetics in humans. J. Gerontol. 41, 319-324, 1986.

ZUMOFF, B., STRAIN, G.W., KREAM, J., et al. Age variation of the 24-hour mean plasma concentrations of androgens, estrogens, and gonadotropins in normal adult men. J. Clin. Endocrinol. Metab. 54, 534-538, 1982 . 